\title{
Studies on Dissolution Behaviour of Nanoparticulate Curcumin Formulation
}

\author{
R. Ravichandran \\ Regional Institute of Education (NCERT), Bhopal, India \\ Email: ravincert@gmail.com
}

Received October 22, 2012; revised November 25, 2012; accepted December 10, 2012

\begin{abstract}
Curcumin is the main biologically active phytochemical compound of turmeric that has been widely used by ancient cultures throughout Asia. However the dissolution rate limited absorption and pre-absorption degradation limits its use as a potential therapeutic. In this study an attempt has been made to overcome the above limitations by curcumin delivery through nanotechnology. Nanocurcumin solid dosage formulations were prepared and studied for its dissolution behaviour. Considerable improvement in the dissolution behavior was observed in the drug nanocrystal-loaded solid dosage forms. This is expected to enhance the bioavailability of poorly soluble medicinal herbs such as turmeric in the body.
\end{abstract}

Keywords: Curcumin; Nanocrystal; Dissolution Evaluation

\section{Introduction}

Curcumin (1,7-Bis(4-hydroxy-3-methoxy phenyl)-1-6heptadiene-3,5-dione) (Figure 1) is a naturally occurring golden yellow compound found as the main constituent in the rhizome of the plant Curcuma longa grown in tropical Southeast Asia [1]. Curcumin is the principal curcuminoid of no change the popular Indian spice turmeric, which is a member of the ginger family Zingiberaceae. It is estimated that $2 \%-5 \%$ of the turmeric is curcumin. Today, India is the primary exporter of turmeric (known as haldi in Hindi, India). For centuries, turmeric has been used as a spice, in preservation of food through its antioxidant mechanism, colouring agent in Indian food, as a yellow dye for textiles and, as well as a therapeutic agent in traditional Indian medicine to treat a wide variety of ailments, with no known side effects $[2,3]$. Curcumin, which gives the yellow colour to turmeric, was first isolated in 1815, and its structure as diferuloylmethane was determined in 1910 by Kazimierz Kostanecki, J. Miłobędzka and Wiktor Lampe. Turmeric has been used historically as a component of Indian Ayurvedic medicine since $1900 \mathrm{BCE}$ to treat a wide variety of ailments including those of the skin, pulmonary, and gastrointestinal systems, aches, pains, wounds, sprains, and liver disorders [4]. Extensive research in the latter half of the 20th century has identified curcumin as responsible for most of the biological activity of turmeric [5]. Curcumin has been shown to exhibit anti-oxidant, anti-inflammatory, anti-viral, anti-bacterial, anti-fungal, anti-cancer hyperlipidemic, woundhealing and hepatoprotective activities and thus has a potential against various malignant diseases, diabetes, allergies, arthritis, Alzheimer's disease, and other chronic illnesses [6]. These effects are mediated through the regulation of various transcription factors, growth factors, inflammatory cytokines, protein kinases, and other enzymes [7]. Considering the recent scientific bandwagon that multitargeted therapy is better than monotargeted therapy for most diseases, curcumin can be considered an ideal "Spice for Life" [8]. The pharmacological efficacy of curcumin makes it a potential compound for treatment and prevention of a wide variety of human diseases [9]. In addition, it is extremely safe upon oral administration even at very high doses, as proven in various animal models or human studies [10]. In spite of this, curcumin has not yet been approved as a therapeutic agent, and the relative bioavailability of curcumin has been highlighted as a major problem for this [11].

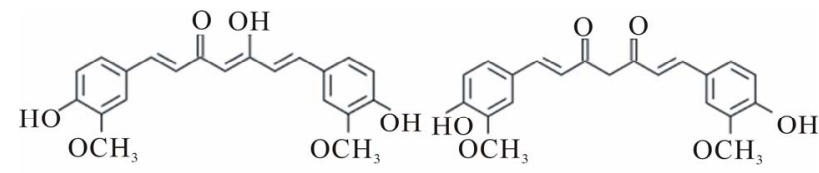

Figure 1. Chemical structure of curcumin: (1E,6E)-1,7-bis (4-hydroxy-3-methoxyphenyl)-1,6-heptadiene-3,5-dione Molecular formula $\mathrm{C}_{21} \mathrm{H}_{20} \mathrm{O}_{6}$; Molar mass $368.38 \mathrm{~g} / \mathrm{mol}$; Melting point $183^{\circ} \mathrm{C}(361 \mathrm{~K})$; Exhibit keto-enol tautomerism and the enol form is more energetically stable in the solid phase and in solution $\sim 95 \%$. 
Curcumin is insoluble in water under acidic or neutral conditions $(0.1 \mathrm{mg} / \mathrm{ml})$ but dissolves in alkaline environment $(3 \mathrm{mg} / \mathrm{ml})$. Curcumin is hydrophobic in nature and frequently soluble $(1 \mathrm{mg} / \mathrm{ml})$ in acetone $(20 \mathrm{mg} / \mathrm{ml})$, ethanol, dimethylsulfoxide, dimethyl formamide and oils. Curcumin is highly unstable undergoing rapid hydrolytic degradation in neutral or alkaline conditions to feruloyl methane and ferulic acid [12]. It is reported to be stable below $\mathrm{pH}$ 6.0. Thus, the use of curcumin is limited by its poor aqueous solubility in acidic or neutral conditions and instability in alkaline $\mathrm{pH}$. This leads to slow dissolution and reduced bioavailability to incomplete oral absorption $60 \%$, such that therapeutic effects are essentially limited to the tubular lower GI tract (i.e., colorectum) [13-15]. Phase I clinical trials have shown that curcumin is safe even at high doses (12 g/day) in humans but exhibits poor bioavailability [16]. Major reasons contributing to the low plasma and tissue levels of curcumin appear to be due to poor absorption, rapid metabolism, and rapid systemic elimination. To improve the bioavailability of curcumin, numerous approaches have been undertaken. These approaches involve, first, the use of adjuvant like piperine that interferes with glucuronidation [17]; second, the use of liposomal curcumin [18]; third, curcumin nanoparticles [19]; fourth, the use of curcumin phospholipid complex [20]; and fifth, the use of structural analogues of curcumin (e.g., EF-24) [21]. The latter has been reported to have a rapid absorption with a peak plasma half-life. Despite the lower bioavailability, therapeutic efficacy of curcumin against various human diseases, including cancer, cardiovascular diseases, diabetes, arthritis, neurological diseases and Crohns disease, has been documented. Enhanced bioavailability of curcumin in the near future is likely to bring this promising natural product to the forefront of therapeutic agents for treatment of human disease.

The development of a delivery system that can enable parenteral administration of curcumin in an aqueous phase medium will significantly harness the potential of this promising anti-cancer agent in the clinical arena. Several approaches to enhance the solubility of Curcumin such as chemical derivatisation, complexation or interaction with macromolecules e.g. gelatin, polysaccharides, protein, and cyclodextrin have been reported but not successful yet in practical utility [22]. Nanonisation is one of the successful methods in improving drug dissolution. In the resent years there have been considerable interests in developing nanoparticles as effective drug delivery system that can effectively deliver the drug to a target site and increase the therapeutic effect, while minimizing any side effects $[23,24]$. We attempted to develop Curcumin nanocrystal as an innovative solution to overcome the oral no change bioavailability problem of Curcumin.

Since a decade the area of oral nanoparticulate drug delivery systems has received considerable attention $[23,24]$. Nanoparticulate systems studied include polymeric, solid lipid, liposomes, niosomes, micelles, microemulsions, submicron emulsions and drug nanoparticles. Nanonization is gaining commercial importance with more products seen in the market [25-29]. Rapamune containing Sirolimus (Wyeth) and Emend containing Fenofibrate (Merck) are commercially available in the market with drug as nanocrystals. Many other products are in pipeline at different phases of clinical trials [30]. Drug nanoparticles or drug nanosuspensions consist of drug particles between 200 and $500 \mathrm{~nm}$ stabilized with/without additives [30]. Nanonization helps in significant improvement of pharmacokinetic parameters of poorly bioavailable drugs [31-34]. Unlike other nano systems this dosage form contains surfactants at very low concentrations. Curcumin has dissolution rate limited bioavailability and therefore formulating as nanosuspension might improve its dissolution rate thereby enhancing oral bioavailability $[2,5,9,10]$. The ultimate goal of this research was to produce a greater improvement in the dissolution and bioavailability of curcumin through nanoparticulate formulations. In previous paper we reported the preparation and characterisation of Curcumin nanosuspension [35]. The present paper deals with the preparation of a new Curcumin nanocrystal capsule formulation for oral administration along with the dissolution behaviour.

\section{Materials and Methods}

\subsection{Materials}

Curcumin was a gift sample from Indsaff Inc., Bhubaneswar, India. Curcumin nanosuspensions were stabilized by Polyvinyl alcohol (PVA, molecular weight 90,000) and sodium dodecyl sulfate. Double-distilled water was used as dispersion medium. The other chemicals were of analytical reagent grade (SRL, Mumbai, India).

\subsection{Preparation of Curcumin Nanosuspensions}

The Curcumin nanosuspension (Cur-NS) was produced via high pressure homogenization in pure water using a Micron Lab 40 at room temperature, applying 20 homogenization cycles at 1500 bar (equal to $150,000 \mathrm{kPa}$ and 21,756 psi) from spray dried material.

\subsection{Spray-Drying}

Spray-drying was employed to obtain freely flowable Curcumin nanocrystal powder. For that Curcumin nanosuspensions, having a drug concentration of $10 \%(\mathrm{w} / \mathrm{w})$, were dried with a Mini Spray-dryer B-190. The mini Spray-dryer B-190 was set with regard to temperature inlet $\left(110^{\circ} \mathrm{C}-100^{\circ} \mathrm{C}\right)$, outlet $\left(74^{\circ} \mathrm{C}-76^{\circ} \mathrm{C}\right)$ and air vol- 
ume $(600 \mathrm{l} / \mathrm{h})$. The spray-dried Curcumin nanocrystals were directly collected after the process.

\subsection{Particle Size Analyses}

Photon correlation spectroscopy (PCS) (Zetasizer Nano ZS) and laser diffraction (LD) (Coulter LS230) were employed to determine the particle size. PCS measurements were performed at $20^{\circ} \mathrm{C}$ and each sample was analysed three times. PCS yields the intensity weighted mean diameter of the bulk population (z-average, measuring range: $3 \mathrm{~nm}-3 \mu \mathrm{m}$ ) and the polydispersity index (PI) as measures for the width of the size distribution. The PI ranges from zero (monodisperse particles) to 0.500 (broad distribution), values above 0.5 do not allow allocation of a logarithmic normal distribution to the PI. LD was repeated three times and the results were calculated as volume size distribution using Mie theory with the optical parameters 1.593 for the real refractive index and 0.01 for the imaginary refractive index. As characterisation parameters the diameters $10 \%, 50 \%, 90 \%$ and $99 \%$ were used. For example diameter $99 \%$ means that $99 \%$ of the particles are below the given size value.

\subsection{Preparation of the Capsule and Drug Release}

Formulations of the Curcumin capsules can be seen in Table 1. Curcumin was admixed to the capsule excipients by a tumbler (Turbula, Basel). The mixed powder was filled into hard gelatin capsule No. 2 using simple filling capsule equipment for lab scale. The final product of the capsules was collected and immediately transferred into dry plastic containers and tightly sealed.

The dissolution test of the capsules was performed using a USP XXIV rotating paddle apparatus with a Pharmatest PTW SIII (Pharma Test, Hamburg, Germany) at $37^{\circ} \mathrm{C}$ and a rotating speed of $50 \mathrm{rpm}$ in $900 \mathrm{ml}$ of medium. Capsules were held to the bottom of the vessel using copper sinkers. Samples were analyzed for drug concentration using HPLC.

\subsection{HPLC Analysis of Curcumin}

A validated sensitive and selective high-performance liquid

Table 1. The formulations of curcumin capsules.

\begin{tabular}{cccccc}
\hline \multirow{2}{*}{ Formulation } & \multirow{2}{*}{$\mathrm{NC}(\mathrm{mg})$} & $\mathrm{MC}(\mathrm{mg})$ & \multicolumn{4}{c}{ Excipient $(\mathrm{mg})$} \\
\cline { 4 - 6 } & & & $\mathrm{Lc}$ & $\mathrm{AV}$ & $\mathrm{Mg}$ \\
\hline A & 500 & - & 15 & 190 & 1 \\
B & - & 500 & 15 & 190 & 1 \\
Market & - & 500 & - & - & - \\
\hline
\end{tabular}

$\mathrm{NC}=$ Curcumin nanocrystal, $\mathrm{MC}=$ Curcumin microcrystal; $\mathrm{Mg}=$ Magnesium stearate, $\mathrm{AV}=$ Avicel $\mathrm{PH} 102, \mathrm{Lc}=$ Lactose, Market $=$ Marketed capsule. chromatography (HPLC) no change method using UV detection was used for the determination and quantification of curcumin. The HPLC system consisted of a Shimadzu LC 6A HPLC instrument equipped with a solvent delivery pump, a Rheodyne injector valve and a variable wavelength UV detector. The column used was C 18 analytical column $(4.6 \times 250 \mathrm{~mm}$, particle size $5 \mu \mathrm{m})$, with mobile phase consisting of two components: A, 10 $\mathrm{mM}$ ammonium acetate $\mathrm{pH} 4.5$; $\mathrm{B}$, acetonitrile. Initial conditions were $95 \%$ A progressing to $55 \% \mathrm{~A}$ at $20 \mathrm{~min}$ and $5 \% \mathrm{~A}$ at $33 \mathrm{~min}$. The flow rate was maintained at 1 $\mathrm{mL} / \mathrm{min}$ at $45^{\circ} \mathrm{C} \pm 2^{\circ} \mathrm{C}$. The eluate was monitored at 420 $\mathrm{nm}$. Retention time for curcumin was $8 \mathrm{~min}$. Free curcumin is completely insoluble in water therefore the concentration of curcumin was calculated using standard curve of curcumin in ethanol. The data was recorded and calculated using Winchrome software.

\section{Results and Discussion}

\subsection{Curcumin Nanocrystals}

The curcumin nanosuspension on a lab scale is typically produced by pre-milling (with SDS $0.2 \%$ ) followed by high pressure homogenization in pure water using a continuous Micron LAB 40 at room temperature, applying 20 homogenization cycles at 1500 bar. The formulation of curcumin nanosuspension was prepared using Curcumin $10 \%$, Polyvinyl alcohol $2 \%$ and Water $88 \%$. Figure 2 clearly shows the difference between the ordinary suspension and the nanosuspension obtained. Nanonisation renders curcumin completely dispersible in aqueous media. Free curcumin is poorly soluble in aqueous media, and macroscopic flakes can be seen floating in the bottle. In contrast, the equivalent quantity of curcumin nanoparticles is fully dispersible in aqueous media. Figure 3 shows the particles size distribution of curcumin formulations by PCS and LD. The Cur-NS-B had LD particle size distribution of $0.1 \mu \mathrm{m}(<\mathrm{d} 10 \%), 0.2 \mu \mathrm{m}(<\mathrm{d} 50 \%)$, $1.8 \mu \mathrm{m}(<\mathrm{d} 90 \%)$ and $2.8 \mu \mathrm{m}(<\mathrm{d} 99 \%)$. PCS size $306 \mathrm{~nm}$, Zeta potential $(\mathrm{mV})$ of -6.4 in water and -2.7 in original medium. Visual examinatiosn of crystals in nanosuspensions from images of the nanosuspensions from light microscopy and scanning electron microscopy showed

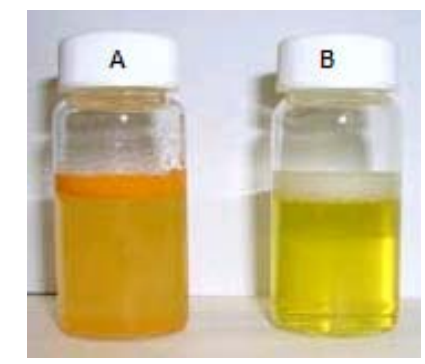

Figure 2. (A) Ordinary suspension and (B) the nanosuspension of curcumin. 


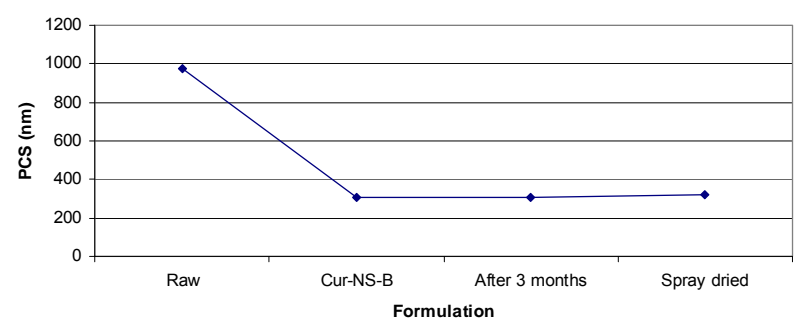

(a)

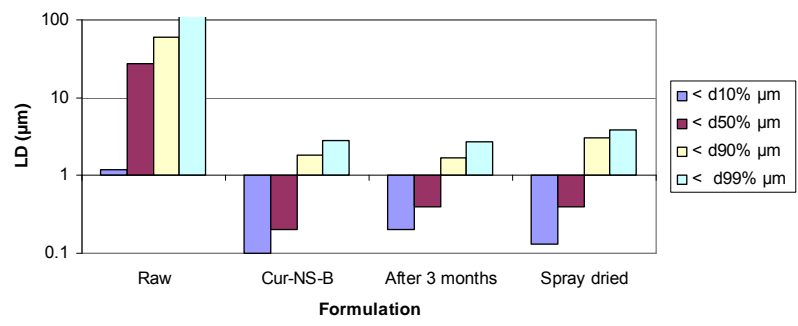

(b)

Figure 3. (a) PCS (above) and (b) LD particles size distribution (below) of curcumin formulations.

fine stable homogeneous distribution. It showed very good physical and chemical stability over 3 and 6 months period respectively. A spray drying process was employed to obtain dried curcumin nanocrystals having good re-dispersability, saturation solubility and dissolution velocity. $\mathrm{LD}$ values were $0.13 \mu \mathrm{m}(<\mathrm{d} 10 \%), 0.4 \mu \mathrm{m}$ $(<\mathrm{d} 50 \%), 3.1 \mu \mathrm{m}(<\mathrm{d} 90 \%)$ and $3.9 \mu \mathrm{m}(<\mathrm{d} 99 \%)$. PCS size was $321 \mathrm{~nm}$ and PI of 0.38 . In general, the saturation solubility of the nanocrystals was distinctly 5 fold higher than for microparticles. The result also showed the superiority of curcumin nanocrystals in dissolution behavior and was in agreement with the Noyes-Whitney equation. According to these results, curcumin nanocrystals are suitable for incorporation into solid dosage form, such as tablets, capsules, pellets etc. The final product of a solid dosage form containing curcumin nanocrystals is evaluated in this work with respect to dissolution testing and compared to marketed dosage forms. This part of the study has been communicated in detail recently.

\subsection{Preparation of Solid Dosage Forms}

Nanosizing refers to the reduction of drug particle size down to the submicron range. While reduction of particle size has been employed in pharmaceutical industry for several decades, recent advances in milling technology and our understanding of such colloidal systems have enabled the production of drug particles of $50-200 \mathrm{~nm}$ size in a reproducible manner. The sub-micron particles are stabilized with surfactants or polymers in nanosuspensions which can be further processed into standard dosage forms suitable for oral administration. These nanosuspensions offer increased dissolution rates for drug compounds and complement other technologies used to enhance the bioavailability of insoluble compounds (BCS Class II and IV), such as solubility enhancers (i.e. surfactants), liquid-filled capsules or solid dispersions of drugs in their amorphous state. Based on the NoyesWhitney principle, reduction in the particle size will increase the dissolution rate due to increased effective particle surface area. This size-dependency comes only into effect for particles of a size below approximately $1 \mu \mathrm{m}$ (submicron particulate) - a phenomenon observed in solid dosage which leads to an increase of the dissolution rate of such fine drug particles $[23,24]$.

\subsection{Formulation}

On the market, no oral curcumin capsule is found in single dose form. Curcumin is normally not combined with any other drugs. CUR- $500^{\circledR}$ capsules compared in this study contain $500 \mathrm{mg}$ curcumin. The formulations of curcumin capsules can be seen in Table 1. Curcumin nanocrystals were admixed to the capsule excipients in a tumbler. On a lab scale, the capsules were prepared using simple capsule filling equipment. The mixed powder was gently poured into capsule no. 2 using the capsule filling equipment. Avicel PH 102, lactose, magnesium stearate are commonly used excipients for capsule fillers. Avicel 102 has good characteristics for a capsule filler, such as excellent flow ability in comparison to Avicel 101. Therefore Avicel PH 102 was mostly chosen as the capsule filler. $\mathrm{Mg}$ stearate was incorporated as lubricant, glidant and anti-adherent. These capsules were furthermore evaluated with respect to their dissolution behavior. The final product of the curcumin nanocrystal-loaded capsules can be seen in Figure 4.

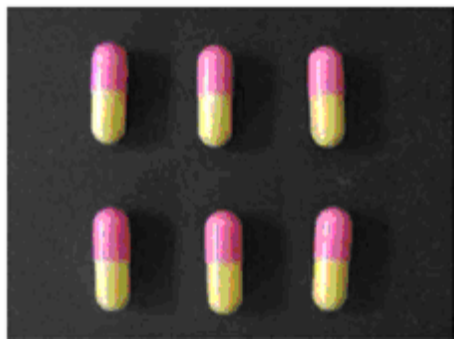

(a)

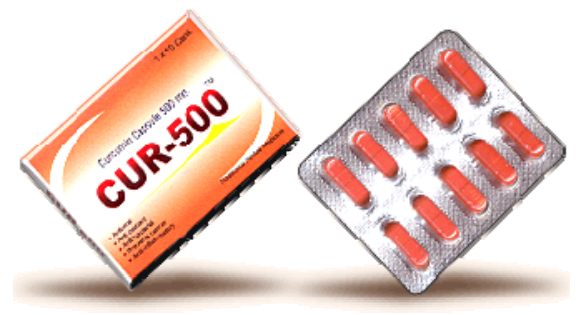

(b)

Figure 4. Curcumin nanocrystal-loaded capsules (a) and marketed capsules (b). 


\subsection{Dissolution Study of Capsules}

In vitro dissolution testing of Curcumin capsules was performed in water and buffer at $\mathrm{pH} 1.2$ and 6.8 , at $37^{\circ} \mathrm{C}$ (Figure 5). The dissolution test was not performed in sink conditions. Therefore at a certain time, release of Curcumin will be constant or further increase little because the saturation solubility is going to be approached. Dissolution velocity of Curcumin from the Curcumin nanocrystal-loaded capsules (nanocrystal capsules) was distinctly improved and superior to that of micro crystals capsules. Within 30 minutes, almost all of Curcumin was dissolved from the nanocrystal capsules (Formulation A) in water and buffer having a $\mathrm{pH}$ of 6.8. However, only $50 \%$ Curcumin was dissolved in buffer having a $\mathrm{pH}$ of 1.2. In contrast, only less than half of Curcumin was dissolved from the microcrystal capsules (Formulation B) in water and in buffer having a $\mathrm{pH}$ of 6.8 , and very little in buffer having a $\mathrm{pH}$ of 1.2.

\subsection{Comparison of Nanocrystal-Loaded Capsules and Marketed Capsules}

Further studies were conducted in order to ascertain the efficacy of Curcumin nanocrystals against the marketed commercial Curcumin capsules. The commercial curcumin products available in the market also contain micro crystals of curcumin but in a typical unknown formulation. In order to make sure that the excipient used in our study does not add to the observed behavior and to have a direct comparison with market product further study was conducted to make certain the efficacy of Curcumin nanocrystals against the marketed commercial capsules. Figure 6 shows the dissolution behaviour of these two products in various medium. Due to the poor aqueous solubility of Curcumin, marketed capsules were unable to release Curcumin similar to microcrystal loaded capsules studied above. In contrast, Curcumin nanocrystals showed an increased kinetic solubility in all the medium studied. Therefore, the dissolve of Curcumin from these capsules was distinctly improved. After the initial nag period, nanocrystals showed near cent percent dissolution in water and at $\mathrm{pH}$ 6.8. At $\mathrm{pH} 1.2$ it showed all most half of dissolution. In contrast the marketed commercial capsules containing Curcumin microcrystals showed very poor dissolution compared to nanocrystals in all the cases studied. Many researchers have evaluated the improved dissolution of Curcumin at $37^{\circ} \mathrm{C}$ in distilled water and other body $\mathrm{pH}$ ranges [36]. The result shows that dissolution of Curcumin was considerably enhanced by solid dispersion of nanoparticles. However, their results did not specifically describe improved dissolution of $\mathrm{Curcu}$ min nanoparticles, instead mentioning only prolonged release of Curcumin from nanoparticles. Dissolution velocity of nanocrystal-loaded capsules is much better than any other techniques. Among factors for consideration,

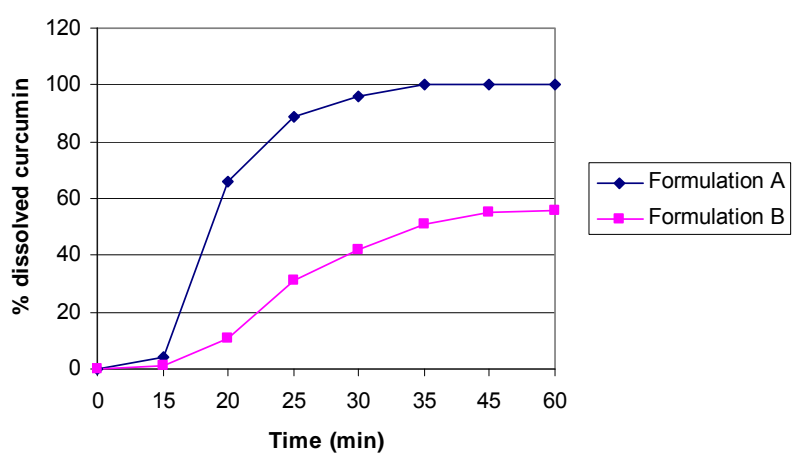

(a)

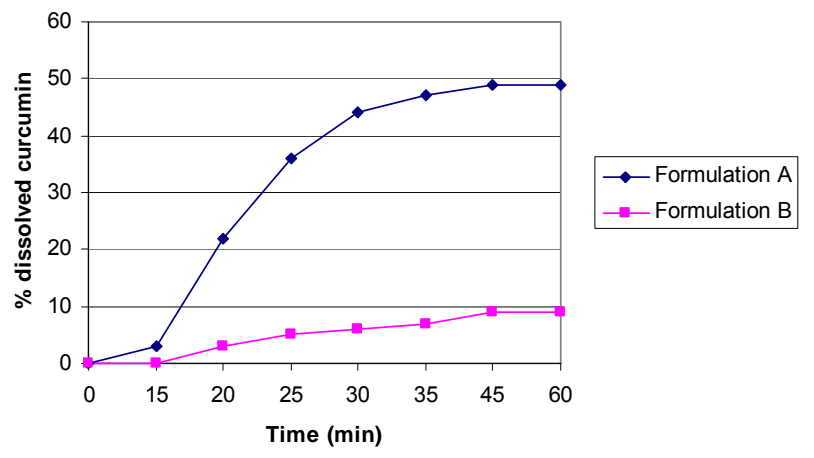

(b)

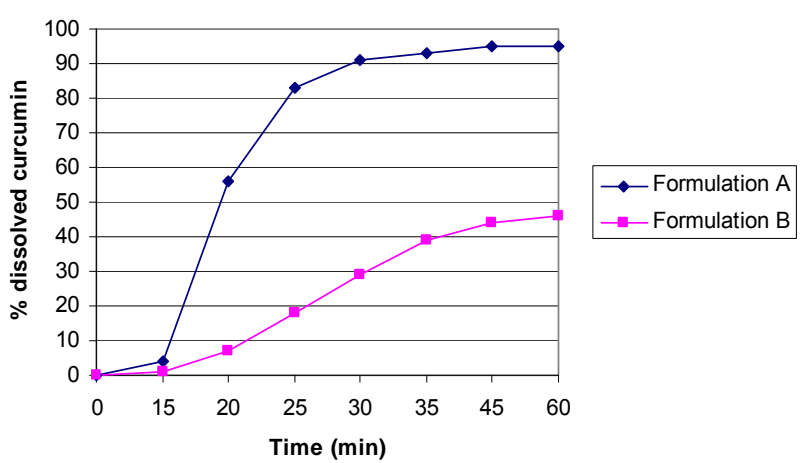

(c)

Figure 5. Percentage of dissolved Curcumin from nanocrystal-loaded capsules (formulation A) compared to microcrystal-loaded capsules (formulation B) in water ((a): Top); buffer at pH 1.2 ((b): Middle) and pH 6.8 ((c): Bottom).

nanocrystals are simpler to manufacture and require less material. Nanotechnology is therefore more effective in increasing solubility and dissolution velocity. Moreover this technology offers cost saving.

The more rapid release of Curcumin from the nanocrystal-loaded capsules is thought to be beneficial since, once being administered orally, the fast dissolved Curcumin may be passively partitioned into the gastrointestinal tract tissues in a shorter time (because of the favorable concentration gradient) resulting into a rapid onset of action and improved bioavailability. Therefore, Curcumin nanocrystal-loaded capsules offer promise for a dosage form with superior physicochemical properties 


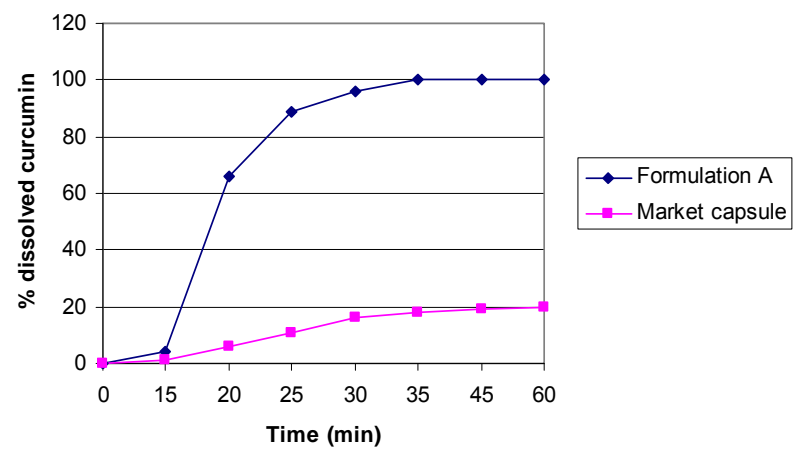

(a)

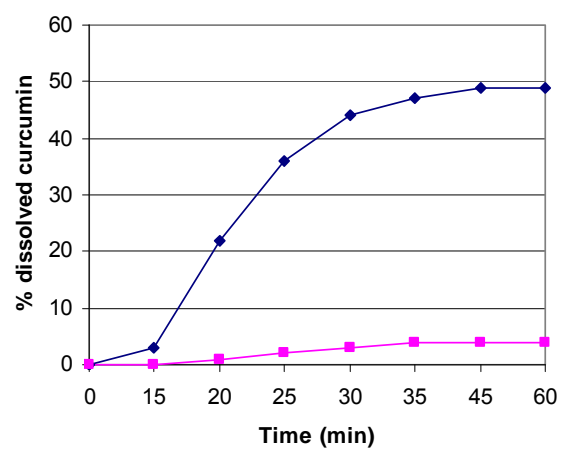

$\rightarrow$ Formulation A $\rightarrow-$ Market capsule

(b)

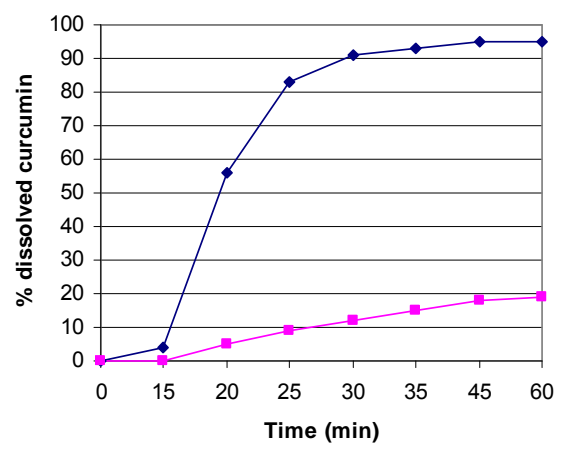

(c)

Figure 6. Percentage of dissolved Curcumin from nanocrystals-loaded capsule in comparison with marketed capsule in water ((a): Top); buffer at pH 1.2 ((b): Middle) and pH 6.8 ((c): Bottom).

that overcomes problem of low bioavailability in the human body.

\subsection{Dissolution Performance}

Compared to microcrystal capsule and marketed capsule, the nanocrystal capsules definitively showed higher levels of percentage dissolved curcumin. In all the dissolution media, nanocrystal capsules dissolved curcumin at distinctly faster rates compared to microcrystals and marketed capsules. Therefore, nanocrystal capsules have superior characteristics to microcrystals and marketed capsules, indicating a major opportunity to enhance the bioavailability of drugs by nanosuspensions for oral ad- ministration, in cases when dissolution is a rate limiting factor in bioavailability in the body like that of curcumin. It is easy to understand that a bio-relevant medium will need a similar surface activity as bio-fluids and hence a study on "In vivo pharmacokinetic evaluation of curcumin nanoformulation with improved bioavailability" was also carried out and is found to yield a similar positive result (communicated).

\subsection{Dissolution Velocity}

An outstanding feature of nanocrystals is the increase in saturation solubility and consequently an increase in the dissolution velocity of the compound. Based on the Noyes Whitney equation [37], this increase in dissolution velocity takes place in addition to the increase caused by the enlargement of the surface area, e.g. exploited in micronized products [38]. By decreasing the particle size (e.g. to the nanometer range), consequently the surface area of the particulate is further increased. In addition, the Noyes-Whitney equation also describes that the dissolution velocity $\mathrm{d} c / \mathrm{d} t$ depends on the concentration gradient $(c s-c x) / h$ (cs is the saturation solubility; $c x$ the equilibrium concentration in the bulk phase and $h$ the diffusional distance) and the Prandtl equation describes that the diffusional distance $h$ is reduced for small particles. Thus, the simultaneous increase in the saturation solubility cs and the decrease in $h$ lead to an increased concentration gradient $(c s-c x) / h$, enhancing the dissolution velocity in addition to the surface effect [39].

An increase in dissolution velocity and also an increase in saturation solubility can also be achieved by changing the crystalline state of the material (e.g. from crystalline to amorphous or partially amorphous). Due to thermodynamic reasons the preservation of the amorphous state is critical; therefore the production of nanocrystals should lead to crystalline particles. The crystalline state of the curcumin nanocrystals investigated in our study remained unchanged (100\% crystalline) upon both, high pressure homogenization and drying process.

\subsection{Perspectives of Drug Nanocrystals for Oral Application}

Recently, the particle size reduction effectiveness of drug substances-loaded tablets on oral bioavailability has been intensively investigated [23]. It has been proven that particle size reduction leads to improved oral bioavailability in the body. Takano et al. have specified that particle size reduction leads to improved dissolution rate and bioavailability [40]. In addition, the rate-limiting steps of oral absorption were simulated. An increase in the dissolution rate and administered dose showed a shift from dissolution rate-limited to solubility limited absorption. In the study in dogs, the particle size reduction of the drugs 
improved the oral absorption [40]. Such studies provide a powerful tool to predict dose linearity and will aid in the development of formulating poorly soluble drugs (Biopharmaceutical Classification System (BCS) class II (as well as IV) drugs).

According to Hintz et al., a computer method has been developed to describe the theoretical dissolution rate of a polydisperse powder under non-sink conditions based on its weight percent particle size distribution. It is shown that finer particles in the size distribution showed an improved dissolution behavior. Moreover the particle size distributions were used to simulate their effect on the amount of drug absorbed orally [41]. Similarly we suggest this promising curcumin capsule dosage form for oral administration. It leads to superior physicochemical properties and should overcome the in vivo absorption problem of the poorly soluble curcumin as class II BCS drug. Figure 7 summarizes the effects on bioavailability enhancement in the gut. Important is that the nanocrystals are released from the capsule or tablet as fine nanocrystals. It could be shown that a slight aggregation does not yet impair the dissolution velocity, but pronounced aggregation will decrease the dissolution velocity strongly [42].

\section{Conclusion}

Nanosuspensions were formulated by high pressure homogenization to overcome problems caused by poor aqueous solubility. Increase in surface area enhances the dissolution rate. Spray dried curcumin nanocrystals prepared by high pressure homogenization technique can be employed to produce solid dosage forms of the drug like capsules. Dried drug nanocrystals offer superior physicochemical properties. Curcumin nanocrystal-loaded capsules can be produced using conventional procedures. From the Noyes-Whitney equation, the increased surface area and saturation solubility due to the decreased radius result in increased dissolution velocity. This phenomenon

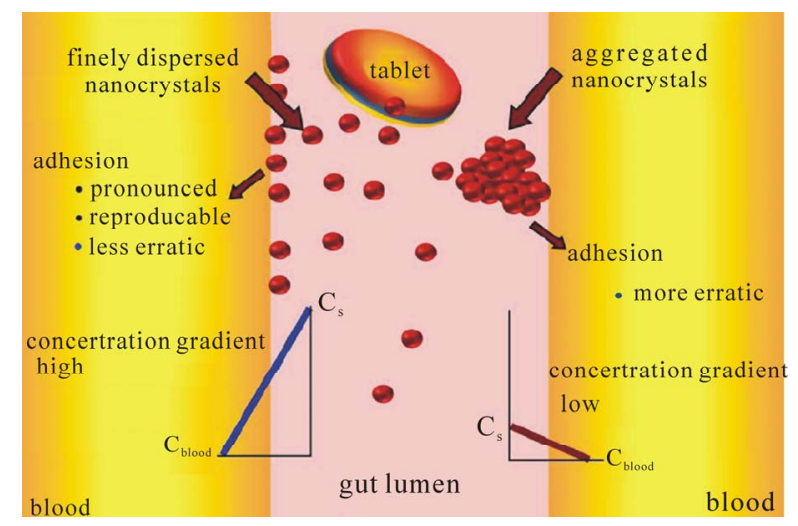

Figure 7. Mechanism of action: finely dispersed nanocrystals versus aggregated nanocrystals (similar to micrometer crystals). was clearly demonstrated by the curcumin nanocrystals. Dissolution velocity of nanocrystal-loaded solid dosage forms was evaluated. Drug nanocrystals were released from the nanocrystal capsules at a faster rate compared to microcrystal capsules or marketed capsules. Dissolution velocity of drug nanocrystals from solid dosage forms was superior compared to micro crystal loaded solid dosage forms and the marketed solid dosage forms (capsules). Improved dissolution behavior in drug nanocrystal-loaded solid dosage forms should lead to better bioavailability of poorly soluble drugs in the body.

\section{Acknowledgements}

Facilities were obtained from Torrent Research Center, Ahmedabad, High Security Animal Disease Laboratory, Bhopal and Central Food Technological Research Institute, Mysore.

\section{REFERENCES}

[1] S. Shishodia, G. Sethi and B. B. Aggarwal, "Curcumin: Getting Back to the Roots," Annals of the New York Academy of Sciences, Vol. 1056, No. 1, 2005, pp. 206-217. doi:10.1196/annals.1352.010

[2] I. Chattopadhyay, K. Biswas, U. Bandyopadhyay and R. K. Banerjee, "Turmeric and Curcumin: Biological Actions and Medicinal Applications," Current Science, Vol. 87, No. 1, 2004, pp. 44-50.

[3] J. A. Duke, "CRC Handbook of Medicinal Spices," CRC Press, New York, 2002, pp. 137-144. doi:10.1201/9781420040487

[4] H. P. T. Ammon and M. A. Wahl, "Pharmacology of Curcumin," Planta Medica, Vol. 57, No. 1, 1991, pp. 1-7. doi:10.1055/s-2006-960004

[5] B. B. Aggarwal, C. Sundaram, N. Malani and H. Ichikawa, "Curcumin: The Indian Solid Gold," Advances in Experimental Medicine and Biology, Vol. 595, 2007, pp. 1-75. doi:10.1007/978-0-387-46401-5 1

[6] H. Hatcher, R. Planalp, J. Cho, F. M. Torti and S. V. Torti, "Curcumin: From Ancient Medicine to Current Clinical Trials," Cellular and Molecular Life Sciences, Vol. 65, No. 11, 2008, pp. 1631-1652. doi:10.1007/s00018-008-7452-4

[7] B. Joe, M. Vijaykumar and B. R. Lokesh, "Biological Properties of Curcumin-Cellular and Molecular Mechanisms of Action," Critical Reviews in Food Science and Nutrition, Vol. 44, No. 2, 2004, pp. 97-111. doi:10.1080/10408690490424702

[8] C. Mancuso and E. Barone, "Curcumin in Clinical Practice: Myth or Reality?" Trends in Pharmacological Sciences, Vol. 30, No. 7, 2009, pp. 333-334. doi:10.1016/j.tips.2009.04.004

[9] R. K. Maheshwari, A. K. Singh, J. Gaddipati and R. C. Srimal, "Multiple Biological Activities of Curcumin: A Short Review," Life Sciences, Vol. 78, No. 18, 2006, pp. 2081-2087. doi:10.1016/j.lfs.2005.12.007 
[10] A. Duvoix, R. Blasius, S. Delhalle, M. Schnekenburger, F. Morceau, E. Henry, M. Dicato and M. Diederich, "Chemopreventive and Therapeutic Effects of Curcumin," Cancer Letters, Vol. 223, No. 2, 2005, pp. 181-190. doi:10.1016/j.canlet.2004.09.041

[11] US Food and Drug Administration. http://www.accessdata.fda.gov/Scripts/cder/DrugsatFDA/

[12] H. H. Tonnesen and J. Karlsen, "Studies of Curcumin and Curcuminoids: V. Alkaline Degradation of Curcumin," Zeitschrift für Lebensmittel-Untersuchung und Forschung, Vol. 180, No. 2, 1985, pp. 132-134.

[13] P. Anand, A. B. Kunnumakkara, R. A. Newman and B. B. Aggarwal, "Bioavailability of Curcumin: Problems and Promises," Molecular Pharmaceutics, Vol. 4, No. 6, 2007, pp. 807-818. doi:10.1021/mp700113r

[14] A. K. Bansal, B. Munjal and S. Patel, "Novel Self-Emulsifying Curcumin Compositions with Enhanced Bioavailability," PCT Filed, No. PCT/IB/2009/005154, 2009.

[15] A. K. Bansal, B. Munjal and S. Patel, "Novel Self Nano Emulsifying Curcumin (Curcuminoids) Composition with Enhanced Bioavailability," Indian Patent No. 1776/DEL/ 2008, 2008.

[16] C. D. Lao, M. T. T. Ruffin, D. Normolle, D. D. Heath, S. I. Murray, J. M. Bailey, M. E. Boggs, J. Crowell, C. L. Rock and D. E. Brenner, "Dose Escalation of a Curcuminoid Formulation," BMC Complementary and Alternative Medicine, Vol. 6, No. 1, 2006, p. 10. doi:10.1186/1472-6882-6-10

[17] G. Shoba, D. Joy, T. Joseph, M. Majeed, R. Rajendran and P. S. Srinivas, "Influence of Piperine on the Pharmacokinetics of Curcumin in Animals and Human Volunteers," Planta Medica, Vol. 64, No. 4, 1998, pp. 353-356. doi:10.1055/s-2006-957450

[18] L. Li, F. S. Braiteh and R. Kurzrock, "Liposome-Encapsulated Curcumin: In Vitro and in Vivo Effects on Proliferation, Apoptosis, Signaling, and Angiogenesis," Cancer, Vol. 104, No. 6, 2005, pp. 1322-1331. doi: $10.1002 /$ cncr. 21300

[19] Bisht, Savita, et al., "Polymeric Nanoparticle-Encapsulated Curcumin ('Cur-NC'): A Novel Strategy for Human Cancer Therapy," Journal of Nanobiotechnology, Vol. 5, No. 3, 2007, p. 3. doi:10.1186/1477-3155-5-3

[20] A. Liu, H. Lou, L. Zhao and P. Fan, "Validated LC/MS/ MS Assay for Curcumin and Tetrahydrocurcumin in Rat Plasma and Application to Pharmacokinetic Study of Phospholipids Complex of Curcumin," Journal of Pharmaceutical and Biomedical Analysis, Vol. 40, No. 3, 2006, pp. 720-727. doi:10.1016/j.jpba.2005.09.032

[21] S. L. Thomas, D. Zhong, W. Zhou, S. Malik, D. Liotta, J. P. Snyder, E. Hamel, and P. Giannakakou, "EF24, a Novel Curcumin Analog, Disrupts the Microtubule Cytoskeleton and Inhibits HIF-1," Cell Cycle, Vol. 7, No. 15, 2008, pp. 2409-2417.

[22] H. Hatcher, R. Planalp, J. Cho, F. M. Torti and S. V. Torti, "Curcumin: From Ancient Medicine to Current Clinical Trials," Journal Cellular and Molecular Life Sciences, Vol. 65, No. 11, 2008, pp. 1631-1652. doi:10.1007/s00018-008-7452-4

[23] R. Ravichandran, "Nanoparticles in Drug Delivery: Po- tential Green Nanobiomedicine Applications," International Journal of Green Nanotechnology: Biomedicine, Vol. 1, No. 2, 2009, pp. B108-B130.

[24] R. Ravichandran, "Nanotechnology-Based Drug Delivery Systems," NanoBiotechnology, Vol. 5, No. 1, 2010, pp. 17-33. doi:10.1007/s12030-009-9028-2

[25] X. Chen, T. J. Young, M. Sarkari, R. O. Williams and K. P. Johnston, "Preparation of Cyclosporine a Nanoparticles by Evaporative Precipitation into Aqueous Solution," International Journal of Pharmaceutics, Vol. 242, No. 1, 2002, pp. 3-14. doi:10.1016/S0378-5173(02)00147-3

[26] R. H. Muller, C. Jacobs and O. Kayser, "Nanosuspensions as Particulate Drug Formulations in Therapy Rationale for Development and What We Can Expect for the Future," Advanced Drug Delivery Reviews, Vol. 47, No. 1, 2001, pp. 3-19. doi:10.1016/S0169-409X(00)00118-6

[27] H. G. Weder and P. van Hoogevest, "Nanosuspensions for Intravenous Administration," US Patent No. 5726164, 1998.

[28] R. H. Muller and C. Jacobs, "Buparvaquone Mucoadhesive Nanosuspensions: Preparation, Optimisation and LongTerm Stability," International Journal of Pharmaceutics, Vol. 237, No. 1, 2002, pp. 151-161 doi:10.1016/S0378-5173(02)00040-6

[29] B. E. Rabinow, "Nanosuspensions in Drug Delivery," Nature Reviews Drug Discovery, Vol. 3, 2004, pp. 785796. doi:10.1038/nrd1494

[30] C. M. Keck and R. H. Muller, "Drug Nanocrystals of Poorly Soluble Drugs Produced by High Pressure Homogenization," European Journal of Pharmaceutics and Biopharmaceutics, Vol. 62, No. 1, 2006, pp. 3-16. doi:10.1016/j.ejpb.2005.05.009

[31] G. G. Liversidge and K. C. Cundy, "Particle Size Reduction for Improvement of Oral Bioavailability of Hydrophobic Drugs: I. Absolute Oral Bioavailability of Nanocrystalline Danazol in Beagle Dogs," International Journal of Pharmaceutics, Vol. 125, No. 1, 1995, pp. 91-97. doi:10.1016/0378-5173(95)00122-Y

[32] J. Jinno, K. Naoki, M. Miyake, K. Yamada, T. Mukia, M. Odomi, H. Togu-chi, G. G. Liversidge, K. Higaki and T. Kimura, "Effect of Particle Size Reduction on Dissolution and Oral Absorption of a Poorly Water-Soluble Drug, Cilostazol, in Beagle Dogs," Journal of Controlled Release, Vol. 111, No. 1-2, 2006, pp. 56-64. doi:10.1016/j.jconrel.2005.11.013

[33] G. G. Liversidge and P. Conzentino, "Drug Particle Size Reduction for Decreasing Gastric Irritancy and Enhancing Absorption of Naproxen in Rats," International Journal of Pharmaceutics, Vol. 125, No. 2, 1995, pp. 309-313. doi:10.1016/0378-5173(95)00148-C

[34] L. Gao, D. Zhang and M. Chen, "Drug Nanocrystals for the Formulation of Poorly Soluble Drugs and Its Application as a Potential Drug Delivery System," Journal of Nanoparticle Research, Vol. 10, No. 5, 2008, pp. 845-862. doi:10.1007/s11051-008-9357-4

[35] R. Ravichandran, "Preparation and Characterisation of Curcumin Nanosuspension for Enhanced Solubility and Dissolution Velocity," International Journal of Nano and Biomaterials, Vol. 3, No. 2, 2010, pp. 153-186. 


$$
\text { doi:10.1504/IJNBM.2010.037803 }
$$

[36] F. Dons, Y. Wang, J. Li and Q. Huang, "Preparation of Curcumin Sub-Micrometer Dispersions by High-Pressure Homogenization," Journal of Agricultural and Food Chemistry, Vol. 58, No. 5, 2010, pp. 2848-2853. doi:10.1021/jf903968x

[37] A. A. Noyes and W. R. Whitney, "The Rate of Solution of Solid Substances in Their Own Solutions," Journal of the American Chemical Society, Vol. 19, No. 12, 1897, pp. 930-934. doi:10.1021/ja02086a003

[38] R. H. Muller, C. Jacobs and O. Kayser, "Nanosuspensions for the Formulation of Poorly Soluble Drugs," In: F. Nielloud and G. Marti-Mestres, Eds., Pharmaceutical Emulsions and Suspensions, Marcel Dekker, New York, 2000, pp. 383-407.

[39] R. H. Muller and A. Akkar, "Drug Nanocrystals of Poorly Soluble Drugs," In: H. S. Nalwa, Ed., Encyclopedia of Nanoscience and Nanotechnology, American Scientific
Publishers, Stevenson Ranch, 2004, pp. 627-638.

[40] R. Takano, K. Furumoto, K. Shiraki, N. Takata, Y. Hayashi, Y. Aso and S. Yamashita, "Rate-Limiting Steps of Oral Absorption for Poorly Water-Soluble Drugs in Dogs; Prediction from a Mini Scale Dissolution Test and a Physiologically-Based Computer Simulation," Pharmaceutical Research, Vol. 25, No. 10, 2008, pp. 2334-2344. doi:10.1007/s11095-008-9637-9

[41] R. J. Hintz and K. C. Johnson, "The Effect of Particle Size Distribution on Dissolution Rate and Oral Absorption," International Journal of Pharmaceutics, Vol. 51, No. 1, 1989, pp. 9-17. doi:10.1016/0378-5173(89)90069-0

[42] C. Keck, A. Fichtinger, H. Viernstein and R. H. Muller, "Oral Drug Nanocrystals-Effect of Potential Aggregation on Bioavailability," Annual Meeting of Pharmaceutical Scientists (AAPS), Baltimore, 2004, p. 2746. 